\title{
Proprioception in Parkinson's disease is acutely depressed by dopaminergic medications
}

\author{
P O’Suilleabhain, J Bullard, R B Dewey
}

\begin{abstract}
Objectives-Impaired proprioception has been previously reported in patients with Parkinson's disease. It was hypothesised that dopaminergic medications transiently depress proprioception, with amplification of adventitious movements as a result. This study tested for effects on proprioception of dopaminergic drugs, and for associations between such effects and drug induced dyskinesias.

Methods-In 17 patients with Parkinson's disease, arm proprioception was tested in the practically defined "off" state, and retested 1 hour after taking levodopa or dopamine agonist. Testing consisted of side to side comparison of elbow angle, matching the contralateral elbow angle, and spatial recall of an unrestrained arm. Results-Proprioception deteriorated as hypothesised, reaching significance by one tailed $t$ test for each of the three tasks. The relative deterioration (and the 95\% lower confidence bound for estimated deterioration) was $31 \%$ (4\%) for side to side elbow comparison, was $27 \%$ (11\%) for accuracy in matching the contralateral elbow angle, and was $11 \%(0 \%)$ for spatial recall. Dyskinetic $(n=6)$ and nondyskinetic $(n=11)$ patients did not differ significantly in these effects on proprioception. Control subjects $(n=6)$ and untreated parkinsonian subjects $(n=5)$ did not significantly differ from the parkinsonian patients in the off state.

Conclusions-Administration of levodopa and dopamine agonists were associated with a modest acute suppression in central responsiveness to joint position. It is speculated that compensatory exaggerated movement could account in part for the phenomenon of drug induced dyskinesias.

(F Neurol Neurosurg Psychiatry 2001;71:607-610)
\end{abstract}

Neurology, University of Texas, Southwestern Medical Center, 5323 Harry Hines

Boulevard, Dallas, TX

75390-9036, USA

P O'Suilleabhain

J Bullard

R B Dewey

Correspondence to: Dr P O'Suilleabhain Padraig.OSuilleabhain@ UTSouthwestern.edu

Received 24 January 2001 and in revised form

11 June 2001

Accepted 26 June 2001 disease receiving levodopa. ${ }^{6-8}$ These authors, and other neuroscientists over the years, have speculated that disruption of sensory modalities such as positional perception may contribute to parkinsonian motor signs such as hypokinesia and abnormal posture. ${ }^{9} 10$ Thinking along the same lines, it occurred to us that diminished proprioception in advanced Parkinson's disease could be a factor in the genesis of levodopa induced dyskinesias. Therefore we hypothesised that joint position sensation becomes phasically diminished during pharmacological peaks of levodopa treatment. The brain, receiving weak positional information, would then facilitate exaggerated or adventitious searching movements in an attempt to keep abreast of the deployment of body parts. This pathophysiology would have some relation with the pseudochorea seen in response to peripheral deafferentation. This study was primarily intended to determine if levodopa and dopamine agonists have acute depressant effects on joint position awareness, and if such effects differ between dyskinetic and nondyskinetic patients.

\section{Subjects and methods}

SUBJECTS

Twenty two patients with Parkinson's disease, Hoehn and Yahr stages I-III, were recruited from our clinic. Ages ranged from 40 to 76 years, with an overall mean (SD) of 62.7 (10.7) years. All except one were right handed. The right side was more affected by the Parkinson's disease in 11 patients, the left more affected in nine, and both sides similarly affected in two Five patients had not yet been treated with dopaminergic drugs. The treated patients were taking levodopa $(n=7)$, a dopamine agonist $(n=2)$ or both levodopa and agonist $(n=8)$. One patient was also taking amantadine. Among the treated patients, 11 had not developed dyskinesias as determined by history and prestudy clinical examinations, and six had developed levodopa induced dyskinesias. Age, sex, and unified Parkinson's disease rating scale motor scores (UPDRS-M) while "off” were not statistically different among the untreated, dyskinetic, and non-dyskinetic patients. Healthy spouses of six patients, aged 59.2 (9.3) years, volunteered as normal controls.

To establish that joint position sensation and cognition were grossly intact, a clinical test of kinaesthesia and the Folstein mini mental state examination were administered to all participants in the "off" state. To test kinaesthesia clinically, the examiner grasped the sides of the middle and distal phalanges and partially flexed or extended the distal interphalangeal 
joint 10 times in succession while the blindfolded subject guessed the direction of joint motion. To be included, subjects needed to detect correctly the directions of at least 16 of 20 joint displacements, and to score 27 or higher (out of 30) on the Folstein examination. The other exclusion criteria were a score of 4 on tremor or rigidity items for either arm on the UPDRS-M, any neurological diagnosis apart from Parkinson's disease, and previous brain surgery. No screened subjects were excluded because of kinaesthesia or Folstein scores. All subjects provided informed consent, and our institutional review board approved the study.

Controls and untreated patients were tested on just one occasion, all in the morning. Treated patients with Parkinson's disease were tested twice, firstly in the practically defined "off" state-that is, in the morning at least 12 hours after the previous dose of medications, then retested one hour after taking 1-1.5 times their usual morning dose of levodopa and/or dopamine agonist. The dose multiple actually given depended on the availability of pills of various dosages. In giving a slightly higher than usual dose, the intent was to compensate for the 12 hour medication free period. Dyskinesias were apparent during the retest period in four of six patients in the dyskinetic group, none severe. Among the 17 treated patients with Parkinson's disease, UPDRS-M improved from 16.6 (SD 7.4) in the practically defined “off" state to 12.9 (SD 5.1) 1 hour after taking dopaminergic medications $(\mathrm{p}=0.01)$.

Proprioception testing consisted of three elements: side to side discrimination of elbow angle, matching the contralateral elbow angle, and attempting to relocate a point in space based on proprioceptive recall.

SIDE TO SIDE ELBOW ANGLE DISCRIMINATION An elbow manipulandum was built with hinged and grooved restraints for both arms mounted on a base. The assemblies for each arm could be moved closer or farther apart depending on the subject's shoulder width. Upper arm and forearm restraints could be adjusted independently using turnbuckles and sliding rails on ball bearings to control their elevation angles and lengths respectively. A mechanical engineering protractor was mounted to read the angle between upper arm and forearm restraints.

The upper arm restraint was set at an angle comfortable for the subject, and the examiner adjusted both elbow angles repeatedly. For each trial, one elbow (the reference elbow) was set to 80 degrees and the other (the test elbow) was set to $76,78,82$, or 84 degrees. The sequence of 48 trials among arms and angles was randomised, and the subject was not aware which was the reference arm. The subject simply indicated which arm was the more flexed. Between trials, the examiner flexed and extended both the subject's elbows by about 10 degrees. The main outcome for this test was the number of incorrect guesses, pooling data from both arms for both 2 degree and 4 degree differences. This measure was selected in advance as the primary outcome for the entire study.

\section{MATCHING ELBOW ANGLE}

The elbow manipulandum was again used. This time the reference arm was set by the examiner to $70,75,80,85,90,95$, or 100 degrees. The test arm was initially placed at 80 degrees, and the blindfolded subject then instructed the examiner to adjust the angle to match the reference elbow. Both arms were tested at these seven angles in random order, with random flexion-extension movements of arms between trials. The angular error for each trial was recorded. The main outcome here was the mean absolute angular error. Bias towards flexion as opposed to extension errors was also analyzed.

\section{PROPRIOCEPTIVE RECALL}

The examiner grasped one of the subject's index fingers and moved it from the ipsilateral shoulder to a point somewhere to the front of the subject. The examiner held the index finger in this test location for 5 seconds, and then returned the finger to the subject's shoulder. The subject immediately tried to return the finger to the test position. The examiner selected 16 different points in space for each arm, choosing two points in each of eight spatial divisions relative to the ipsilateral shoulder: medial or lateral, superior or inferior, and near (less than $15 \mathrm{~cm}$ in front) or far. The shoulder and finger locations were digitised for both test and trial displacements using a magnetic field tracking device (Polhemus Fastrak, Colchester, VT, USA). This apparatus consists of a small sensor with three colocated antennae that was placed on the tip of the index finger using an elastic glove, an identical sensor that was taped to the ipsilateral shoulder, and a magnetic field transmitter mounted close behind the subject. The $\mathrm{x}, \mathrm{y}$, and $\mathrm{z}$ distances of the finger relative to the shoulder were calculated for test and trial displacements. The spatial error, calculated as

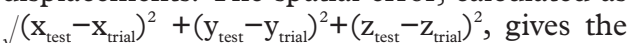
absolute error in three dimensional space. A distance error was also calculated, $/\left(\mathrm{x}_{\text {test }}^{2}+\mathrm{y}_{\text {test }}{ }^{2}+\mathrm{z}_{\text {test }}{ }^{2}\right)-/\left(\mathrm{x}_{\text {trial }}{ }^{2}+\mathrm{y}_{\text {trial }}{ }^{2}+\mathrm{z}_{\text {trial }}{ }^{2}\right)$, negative if the subject undershoots and positive if the subject overshoots the reach. The main outcome for this test was the mean spatial error for 32 attempts.

DATA ANALYSIS

Outcomes for the three Parkinson's disease groups while unmedicated were compared using ANOVA, and as no differences among groups were significant the patients with Parkinson's disease were pooled for $t$ test comparison with the normal controls. Proprioception in the "on" and "off" states were compared with paired $t$ tests, and the differences expressed as percentage change relative to the "off" state. Medication related changes in proprioception were compared between dyskinetic and non-dyskinetic patients using $t$ tests. Comparisons between more parkinsonian and less parkinsonian sides were made among patients 
Table 1 Proprioceptive inaccuracies in healthy controls, never treated PD patients, and both groups of PD patients in the "off" state

\begin{tabular}{lllll}
\hline & $\begin{array}{l}\text { Controls } \\
(n=6)\end{array}$ & $\begin{array}{l}\text { Untreated } \\
(n=5)\end{array}$ & $\begin{array}{l}\text { Dyskinetic } \\
(n=11)\end{array}$ & $\begin{array}{l}\text { Non-dyskinetic } \\
(n=6)\end{array}$ \\
\hline $\begin{array}{l}\text { Side to side elbow comparison: incorrect trials } \\
\text { Matching contralateral elbow: angular error }\end{array}$ & $4.6(3)$ & $6.3(2.5)$ & $5.3(3)$ & $6.3(1.7)$ \\
$\begin{array}{l}\text { Proprioceptive recall: } \\
\quad \text { Spatial error (cm) }\end{array}$ & $3.9(2.7)$ & $3.3(1.2)$ & $3.7(1.8)$ \\
$\quad$ Distance error (cm) & $7.1(2.2)$ & $6.5(1.6)$ & $6.7(1.7)$ & $8.1(3.2)$ \\
\hline
\end{tabular}

Values are group averages (SD).

Table 2 Proprioceptive inaccuracies in the treated PD patients in the "off" compared with the "on" state

\begin{tabular}{llll}
\hline & $\begin{array}{l}\text { Off medications } \\
(n=17)\end{array}$ & $\begin{array}{l}\text { On medications } \\
(n=17)\end{array}$ & p Value \\
\hline $\begin{array}{l}\text { Side to side elbow comparison: incorrect trials } \\
\text { Matching contralateral elbow: angular error }\end{array}$ & $5.7(2.6)$ & $7.4(4.4)$ & 0.03 \\
$\begin{array}{l}\text { Proprioceptive recall: } \\
\quad \text { Spatial error (cm) }\end{array}$ & $7.2(2.4)$ & $8.3(1.5)$ & 0.005 \\
$\quad$ Distance error (cm) & $0.7(1.3)$ & $0.9(1.6)$ & 0.04 \\
\hline
\end{tabular}

Values are group averages (SD).

with Parkinson's disease using a paired $t$ test. Worse proprioception in the "on" as compared with the "off" state, in dyskinetic compared with non-dyskinetic patients, and on the more parkinsonian compared with the less parkinsonian side were hypothesised. One tailed $t$ tests were used to maximise power for those directional effects that make sense in the proposed model.

\section{Results}

PATIENTS WITH PARKINSON'S DISEASE, BOTH UNTREATED AND OFF, COMPARED WITH CONTROLS

Inaccuracies tended to be slightly higher in Parkinson's disease groups than normal controls, but neither the differences between controls and patients with Parkinson's disease, nor the differences among the unmedicated Parkinson's disease groups, reached significance (table 1). Errors were qualitatively similar in each group. For the passive comparison task, incorrect guesses were twice as common for 2 degree interelbow discrepancy compared with 4 degree discrepancy for all groups. For the active matching task, most subjects in all groups tended to have more extension rather than flexion errors - that is, subjects made the test elbow too obtuse more often than too acute. For the proprioceptive recall task, it was noticeable that all subject groups tended to underreach for distance, and that distance errors were considerably smaller than directional errors.

EFFECTS OF MEDICATIONS ON PROPRIOCEPTION On repeated testing 1 hour after medications, there were on average 1.7 more errors for side to side discrimination (table 2). This represents a statistically significant worsening of $31 \%$. The 95\% lower confidence limit for this estimate was a $4 \%$ relative deterioration. The angular error in the matching task had worsened by a mean of 0.9 degrees, a $26 \%$ relative worsening in score, with a lower bound for this estimated deterioration of $11 \%$. The spatial error on the reaching task had worsened by $0.8 \mathrm{~cm}$ on average, an $11 \%$ relative change, with $0.02 \%$ as the lower band of the estimated deterioration. Medication associated worsening of proprioception was similar in dyskinetic and non-dyskinetic patients as determined by the passive and active elbow angle tasks. There was a trend toward more effect among dyskinetic patients in the three dimensional proprioceptive recall: the error was 1.3 (SD $2.7) \mathrm{cm}$ worse in dyskinetic patients on retesting 1 hour after dosing, and 0.6 (SD 1.4) $\mathrm{cm}$ worse in those not prone to dyskinesias. Reclassifying patients by presence or absence of dyskinesias at the time of retesting did not result in significant differences.

COMPARING THE MORE PARKINSONIAN WITH THE LESS PARKINSONIAN ARM

The more parkinsonian arm had mean (SD) spatial error of $6.8(2.0) \mathrm{cm}$ in the "off" state whereas the less affected arm had errors of 7.2 $(2.7) \mathrm{cm}$. Also in the "off" state, the more affected arm was an average $0.8(1.6) \mathrm{cm}$ short for the distance error, whereas the less affected arm was $0.7(1.4) \mathrm{cm}$ short. There were no significant differences between sides for either "off" or "on" states.

\section{Discussion}

Zia et al recently showed abnormal proprioception in treated patients with Parkinson's disease. ${ }^{8}$ These findings complimented previous research which indicated that kinaesthesia is deficient in Parkinson's disease, again primarily among patients receiving dopaminergic medications. ${ }^{5611}$ Although proprioceptive loss may simply be a consequence of neural degeneration, sensory impairment by dopaminergic medications has not been excluded. Indeed, Horak et al examining long loop postural reflexes in Parkinson's disease, found that levodopa reduced the scale of the response. ${ }^{12}$ Abnormal movements in Parkinson's diseasefor example, unaware truncal postural abnormalities and hypokinesis - have for many years been partly attributed to abnormal proprioception. $^{8-10}$ It occurred to us that levodopa might phasically reduce proprioception even further, and that this could contribute to levodopa induced dyskinesias. A related idea was originally proposed by Moore, who found that kinaesthesia in two patients was reduced while dyskinetic compared with while bradykinetic. $^{6}$

In testing for a hypothesised reduction of proprioception by dopaminergic medications, we followed the example of Zia et al in certain respects. ${ }^{8}$ The primary outcome used was bilateral discrimination of the externally imposed elbow angles. A secondary outcome was the accuracy with which the subject could match the contralateral elbow angle, which had been set by the examiner. We used a robust and accurate restraining device, which we think is reliable and not prone to bias. These simultaneous bilateral comparisons are reasonably pure tests of proprioception, avoiding dependence on spatial memory. A minor drawback is the difficulty of assigning sensory impairment to one side more than the other-for example, hypersensitivity on one side and hyposensitivity 
on the other are indistinguishable. We suspected that proprioception and medication response would be worse on the more parkinsonian side, particularly if this side was also more dyskinetic. We therefore decided to also test the accuracy with which the subject could relocate a point in space. This test has some dependence on immediate recall, but it has the advantage of allowing each arm to be tested independently.

In looking for a reversible effect of dopaminergic medications on proprioception, we found a fairly consistent trend for acute worsening of proprioception 1 hour after taking levodopa and/or dopamine agonist. Medications caused $31 \%$ and $26 \%$ worsening of the two tests of elbow proprioception, and $11 \%$ worsening of whole arm spatial recall. The spatial errors were largely directional, with smaller displacement errors, suggesting that the whole arm inaccuracy seen reflected mainly error in shoulder orientation rather than in elbow extension. We considered two factors that could confound interpretation of the results. Dopaminergic medications are sedating to some patients, and if patients were less alert during the retesting, more errors could be expected. Although our patients did not seem drowsy, such an effect cannot be excluded. Secondly, the testing order could have introduced a bias, as the "on" state was always tested second. Boredom or inattention could cause poorer performance on the second series of tests, although subjects had a 1 hour break in between. Although it would have been more rigorous to randomise the sequence of testing, it was more practical for patients to arrive in the practically defined "off" state and complete the evaluations over the next 3 hours allowing for the break. These caveats aside, these results support our hypothesis that dopaminergic medications acutely reduce proprioception in Parkinson's disease, lending some support to our model for dyskinesias. "On" states seem to be associated with relatively lower levels of awareness of joint position, and we suggest that this may temporarily increase adventitious "searching" movements.

We could not confirm a secondary hypothesis that patients prone to dyskinesias had larger fluctuation in proprioception compared with patients in whom dyskinesia had not been documented. Possible explanations include small group sizes, the timing of the retest, and the dose of medication used. The 1 hour time frame for retesting after ingestion may have been too rigid, and a slightly larger than normal morning dose after 12 hours off medication may not have been great enough to maximise the differences among dyskinetic and nondyskinetic patients. It is possible that a larger difference between the groups would be seen after multiple doses of medications.
We found that proprioception, although tending to be poorer, was not statistically different in patients with Parkinson's disease in the off state compared with normal controls. Although not a question of particular interest for this protocol, we reanalysed using the "on" medication results, to see if our data agreed with previous reports. When "on", the 17 patients with Parkinson's disease tended to score worse than controls for each of the tests of proprioception, with differences reaching significance for the elbow discrimination and matching tests but not the spatial recall. By contrast with Sathian et al, we did not find a difference in proprioception between the more parkinsonian and less parkinsonian sides. ${ }^{4}$

Our results add to the decades of research indicating impaired proprioception and kinaesthesia in Parkinson's disease. Similarly to previous authors cited, we suspect that the inaccurate perception of limb position may cause or contribute to abnormal movements in Parkinson's disease. This is the first study to consider the acute effects of dopaminergic medications on proprioception. If our results can be confirmed, they will provide new insight into the mechanisms of medication related movement abnormalities. A contributory role in dyskinesias for phasic pharmacological reduction of proprioception would be further supported if therapies such as amantadine or pallidotomy were shown to have their effects by improving proprioception. In principle, a test could be designed to assess in advance a particular patient's risk of developing dyskinesias by measuring proprioception and its response to dopaminergic medications. Medications and doses could then conceivably be manipulated to minimise the risk of levodopa induced dyskinesias.

1 Parkinson J. An essay on the shaking palsy. London: Sherwood, Neely and Jones, 1817

2 Snider SR, Fahn S, Isgreen WP, et al. Primary sensory symptoms in parkinsonism. Neurology 1976;26:423-9.
sympt

3 Koller WC. Sensory symptoms in Parkinson's disease. NeuKoller WC. Sensory sym
rology 1984;34:957-9.

4 Sathian K, Zangaladze A, Green J, et al. Tactile spatial acuity and roughness discrimination: impairments due to aging and Parkinson's disease. Neurology 1997;49:168-77.

5 Schneider JS, Diamond SG, Markham CH. Parkinson's disease: sensory and motor problems in arms and hands. Neurology 1987;37:951-6.

6 Moore AP. Impaired sensorimotor integration in parkinsonism and dyskinesia: a role for corollary discharges? $\mathrm{F}$ Neurol Neurosurg Psychiatry 1987;50:544-52.

7 Klockgether T, Borutta M, Rapp H, et al. A defect of Klockgether T, Borutta M, Rapp H, et al. A defect of
kinesthesia in Parkinson's disease. Mov Disord 1995;10: kinesthe

8 Zia S, Cody F, O'Boyle D. Joint position sense is impaired by Parkinson's disease. Ann Neurol 2000;47:218-28.

9 Dinnerstein AJ, Frigysei T, Lowenthal M. Delayed feedback as a possible mechanism in parkinsonism. Percept Mot Skills 1962;15:667-80

10 Martin PM. The basal ganglia and posture. London: Pitman, 1967.

11 Jobst EE, Melnick ME, Byl NN, et al. Sensory perception in Parkinson disease. Arch Neurol 1997;54:450-4.

12 Horak FB, Frank J, Nutt J. Effects of dopamine on postural control in parkinsonian subjects: scaling, set, and tone. $\mathcal{f}$ Neurophysiol 1996;75:2380-96. 